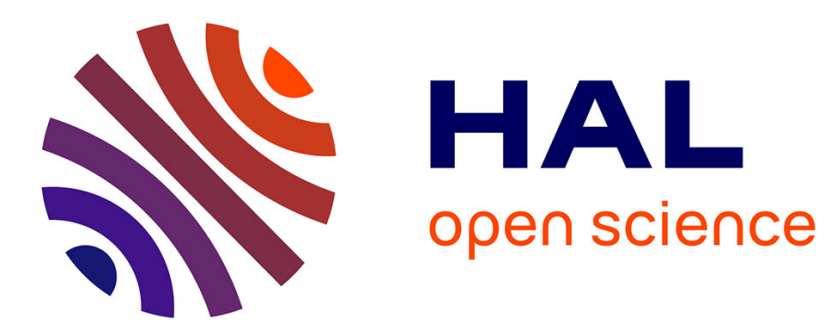

\title{
ETUDE STRUCTURALE DES VERRES ALUMINOFLUOROBERYLLES
}

\author{
Jean-Jacques Videau, Josik Portier, B. Piriou
}

\section{To cite this version:}

Jean-Jacques Videau, Josik Portier, B. Piriou. ETUDE STRUCTURALE DES VERRES ALUMINOFLUOROBERYLLES. Journal de Physique Colloques, 1982, 43 (C9), pp.C9-223-C9-229. 10.1051/jphyscol:1982940 . jpa-00222469

\section{HAL Id: jpa-00222469 https://hal.science/jpa-00222469}

Submitted on 1 Jan 1982

HAL is a multi-disciplinary open access archive for the deposit and dissemination of scientific research documents, whether they are published or not. The documents may come from teaching and research institutions in France or abroad, or from public or private research centers.
L'archive ouverte pluridisciplinaire HAL, est destinée au dépôt et à la diffusion de documents scientifiques de niveau recherche, publiés ou non, émanant des établissements d'enseignement et de recherche français ou étrangers, des laboratoires publics ou privés. 
JOURNAL DE PHYSIQUE

Colloque C9, supplément au n²1, Tome 43, décembre 1982

page $c 9-223$

\title{
ETUDE STRUCTURALE DES VERRES ALUMINOFLUOROBERYLLES
}

\author{
J.J. Videau, J. Portier*et B. Piriou** \\ "Laboratoire de Chimie du Solide du CNRS, 351 cours de la Libération, \\ 33405 Talence Cedex, France \\ * Laboratoire des Eléments de Transition dans les Solides du CNRS, \\ 1. place A.Bmiand, 92190 Meudon-Bellevue, France
}

\begin{abstract}
Résumé. - Des verres aluminofluoroberyllés (ABF) ont été obtenus dans le systēme quaternaire $\mathrm{BaF}_{2}-\mathrm{CaF}_{2}-\mathrm{AlF}_{3}-\mathrm{BeF}_{2}$. Leur structure a été étudiée par PPE, FLN et par spectroscopie Raman. Dans ces verres trois types de groupements formateurs sont présents: $\left(A 1 F_{6}\right),\left(A 1 F_{4}\right)$ et $\left(B e F_{4}\right)$. Des chaines $\left(A 1 F_{5}\right)_{r}$ sont pontées par des tẻtraèdres $\left(B e F_{4}\right)$ et $\left(A 1 F_{4}\right)$ en fajble quantité. Les têtraèdres $\left(B_{F_{4}}\right)$ sont présents sous forme d'espèces plus ou moins polymérisêes $\left(B_{n} F_{3 n+1)}(n+1)\right.$. Ces verres doivent être distingués des matériaux riches en $\mathrm{BeF}_{2}(\mathrm{BF})$ dont le réseau formateur homologue de celuj de la silice vitreuse est constitué de tétraēdres $\left(B_{e} F_{4}\right)$ liés par leurs sommets.
\end{abstract}

Abstract. - Aluminium fluoroberyllate glasses (ABF) obtained in the vitreous state from the quaternary system $\mathrm{BaF}_{2}-\mathrm{CaF}_{2}-\mathrm{AlF}_{3}-\mathrm{BeF}_{2}$ have been studied by EPR, FLN and by Raman spectroscopy. In these glasses, three types of network forming groups are present: $\left(A 1 F_{6}\right),\left(A 1 F_{4}\right)$ and $\left(B e F_{4}\right) \cdot\left(A 1 F_{5}\right)_{n}$ chains are cross-linked with $\left(\mathrm{ATF}_{4}\right)$ in smal1 amount and with (BeF 4 ) tetrahedra. These fluoroberyllate tetrahedra are present as a more or less polymerised species $\left(\right.$ Be $\left._{n} F_{3 n+1}\right)(n+1)$. These ABF glasses must be distinguished from those $r i c h$ in $\mathrm{BeF}_{4}$ groups. The network of fluoroberyllate glasses is constituted of cornerlinked $\left(\mathrm{BeF}_{4}\right)$ tetrahedra.

1. Introduction. - Des études récentes nous ont permis de mettre en évidence de nouveaux verres aluminofluorobéryllës dësignés sour le terme "verre ABF". Les études optiques de ces verres dopés au néodyme ont montré qu'ils possêdajent des caractéristiques optiques supérieures aux matrices vitreuses fluorobéryllées (BF) antérieurement étudiées (1). En conséquence, ces verres riches en fluorure d'aluminium pouvaient posséder une structure différente ce celle des verres fluorobéryllés classiques. I1 nous a donc paru intéressant d'en entreprendre l'étude structurale en nous appuyant sur des techniques telles que la R.PE, la "Fluorescence Line Narrowing" (FLN) couplée à la spectroscopie résọlue dans le temps (S.R.T.) et 1a diffusion Raman, mëthode très appropriẻe pour mettre en évidence les divers motifs structuraux constituant ce type de matêriaux.

2. Synthèse et domaine vitreux. - Des mëlanges de $\left(\mathrm{NH}_{4}\right)_{2} \mathrm{BeF}_{4}, \mathrm{ATF}_{3}$, CaF 2 et $\mathrm{BaF}_{2}$ ont étê prêparés. Après dëcomposition de $\left(\mathrm{NH}_{4}\right)_{2} \mathrm{BeF}_{4}$ le mélange est portè à la fusion, homogénéisé puis soit coulé dans des moules de laiton préchauffés, soit trempé à l'eau. Dans le premier cas les pièces de verres sont recuites puis refroidies très lentement.

L'étude du domaine vitreux dans le système quaternaire $\mathrm{BaF}_{2}-\mathrm{CaF}_{2}-\mathrm{AlF}_{3}-\mathrm{BeF}_{2}$ est donnée à la figure 1 sous la forme de cinq coupes ( $a, b, c, d$, e) à l'échelle du têtraèdre régulier pour cinq différentes teneurs en BeF 2 . Les plus vastes domaines (traits pleins) correspondent aux limites de vitrification observées lorsque le verre est préparé par trempe à l'eau. Les domaines olus restreints inclus dans les précédents (traits pointillês) correspondent à des ëchantilions êlaborés par coulée en moule préchauffé. Cn remarque que la surface occupée nar ces derniers passe par une valeur maximale pour un taux de BeF 2 de 30 mole $\%$ environ. 

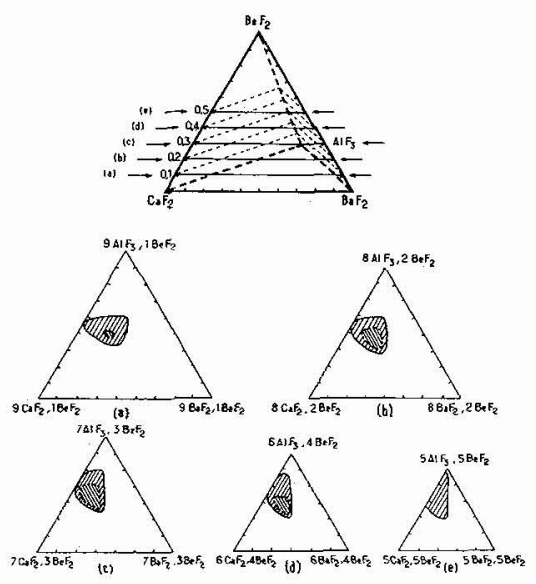

Fig. 1 - Domaines vitreux dans le système quaternaire $\mathrm{BaF}_{2}-\mathrm{CaF}_{2}-\mathrm{AlF}_{3}-\mathrm{BeF}_{2}$ pour cinq coupes différentes, parallèles au plan de base $\mathrm{BaF}_{2}-\mathrm{CaF}_{2}-$ $\mathrm{A}^{1} \mathrm{~F}_{3}$. Les domaines indiquês sont relatifs soit à une trempe à l'eau (traits pleins), soit à une coulée en moule préchauffé (pointillēs).

3. RPE. - L'étude par RPE a porté sur le verre de formule $\left.\mathrm{Ba}_{15} \mathrm{Ca} 28 \mathrm{~A}\right]_{30} \mathrm{Be}_{27} \mathrm{~F}_{230}$ à concentration variable de $\mathrm{Mn}^{2+}(0,15$ mole $\%, 0,50$ mole $\%, 1$ mole $\%)$ introduit sous forme de $\mathrm{MnF}_{2}$.

3.1. Résultats expérimentaux. - La figure 2 représente les spectres obtenus à partir d'un appareil BRUKER ER 200 tt fonctionnant en bandes $\times(9,75 \mathrm{GHz})$ à température ambiante pour les trois compositions étudiées.

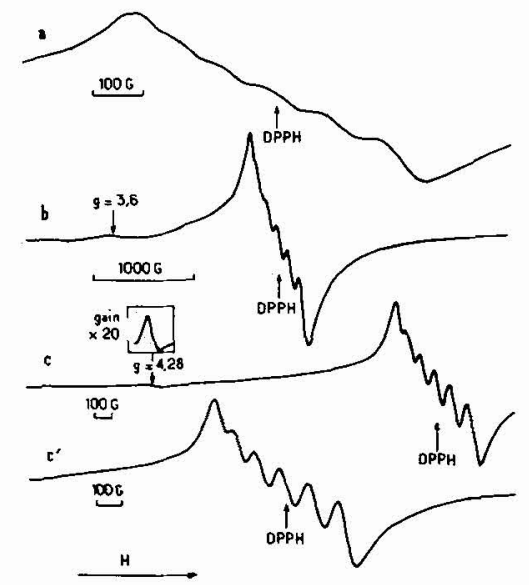

Fig. 2 - Spectres RPE de verres $A B F$ dopés đ̀ $\mathrm{MnF}_{2}$

a -1 mole $\%$

b $-0,5$ mole $\%$

c et $c^{\prime}-0,15$ mole \%

Pour une concentration molaire de $1 \%$ le spectres est constitué d'un massif relativement mal rësolu. Il correspond à la superposition de raies hyperfines ; l'êlargissement de celles-ci êtant due à une contribution dipolaire (interaction entre jons manganèse). Le centre du spectre correspond à une valeur de g très proche ¿́e 2,00 . Pour un dopage de 0,5 mole $\%$ le spectre centré sur $g \simeq 2,00$ est partiellement résolu et il y a apparition des 6 raies de la structure hyperfine. Le calcul donne une valeur de A (paramètre de couplage hyperfin) $\simeq 97 \mathrm{G}$. Dans $1 \mathrm{a}$ portion du spectre obtenue pour des champs plus faibles, on remarque un massif dont le maximum est situé vers une valeur de $g_{\text {eff }} \simeq 3,6$. Pour la concentration la plus 
faible en manganèse $(0,15$ mole \%), une meilleure résolution est obtenue pour le spectre centré sur $\mathrm{g}=2$. La valeur de $\mathrm{A}$ varie peu : $98 \mathrm{G}$. Dans la partie du spectre à champ faible on constate 7 'apparition d'une raie centrée sur geff $=4,28$, 7 arge de $70 \mathrm{G}$ environ et non résolue (pas de structure hyperfine).

3.2. Interprétation et discussion. - Le spectre centré sur $\mathrm{g}=2$ correspond à celui d'un ion Mn2t dans un site dont les termes D et $E$ de 1 'hamiltonien sont traitês comme perturbation de l'interaction Zeeman. Son importance montre que la majeure partie des jons $\mathrm{An}^{2+}$ introduits adoptent une symétrie cubique. En comparant Tes rayons ioniques de $\mathrm{Ca}^{2+}, \mathrm{Mn}^{2+}$ et $\mathrm{Al}^{3+}$ et en tenant compte de $1 \mathrm{a}$ valeur importante de A qui dénote une forte ionicité de la liaison avec les. ligandes, on serait tenté de penser que les ions $M n^{2+}$ se substitueraient en majorité aux ions $\mathrm{Ca}^{2+}$. Les valeurs de geff correspondant à des champs plus faibles indiquent que leur faible quantité d'ions. $\mathrm{Mn}^{2+}$ se placent dans d'autres sites relativement distordus. Geff $=4,28$ révēle pour ceux-ci une forte distortion rhombique $\left(C_{2 v}\right)$ correspondant à une valeur importante de $E(D=0)(2,3)$. Le massif correspondant à $\mathrm{g}=3,6$ provient de la distribution aleatoire des ions $\mathrm{Mn}^{2+}$ dans des sites $C_{2 v}$ comme l'avaient déjã observé GRISCOM D.C. et al dans les vèrres boratés (3).

4. FLN et spectroscopie résolue dans ie temps. - L'étude par FLN et SRT, effectuée à $20 \mathrm{~K}$, a norté sur le verre de composition $\mathrm{B} a{ }_{15} \mathrm{Ca}_{27} \mathrm{~A}_{1} \mathrm{~B}_{35} \mathrm{Be}_{25} \mathrm{~F}_{235}$. I1 est dopé avec 1 mole \% de EuF 3 . Le dispositif expérimental est identique à celui qui a êté utilisé lors d'études précédentes ( 4 ).

4.1. Résultats expérimentaux. - Les excitations sélèctives d'énergie croissante ont été faites dans la bande d'absorption relative à la transition dipolaire électrique ${ }^{5} \mathrm{D}_{0} \rightarrow 7 \mathrm{~F}_{0}$ :

Nous avons enregistré les spẹctres d'émisssion qui en résultaient pour les transitions non résonnantes $5 D_{0} \rightarrow F_{1}$ et ${ }^{5} D_{0} \rightarrow{ }_{F} F_{2}$ (fig. 3). La courbe (b) fait apparâ̂tre une bande étroite vers $5900 \AA$, composée de troìs raies fines correspondant à la transition ${ }^{5 D_{0}} \rightarrow 7_{F_{1}}$, ainsi qu'une bande non résolue, centrée à $6150 \AA$, relative à la transition ${ }^{5} \mathrm{D}_{0} \rightarrow 7_{\mathrm{F}}$. Au fur et à mesure que 1 'énergie d'excitation s'élève, les raies de la transition ${ }^{5 D_{0}} \rightarrow{ }^{7} F_{1}$ deviennent plus intenses tout en restant

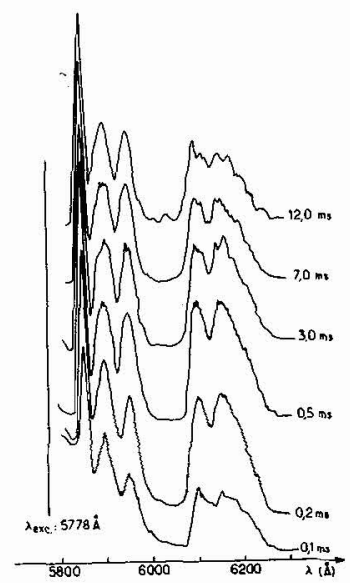

Fig. 4 - Spectres d'ëmission de $\mathrm{Eu}^{3+}$, pour le verre $A B F$ à $20 \mathrm{~K}$, résolus dans le temps pour une excitation unique $\left(\lambda_{\text {exc. }}=5778 \AA\right)$.

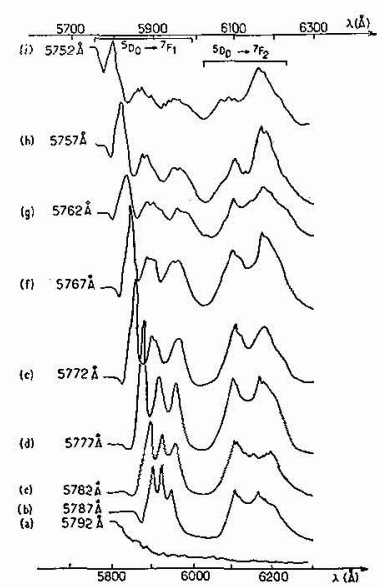

Fig. 3- spectres d'émission de $\mathrm{Eu}^{3+}$ pour le verre $A B F$, étudié à $20 \mathrm{~K}$ $(t=0,5 \mathrm{~ms})$, en fonction de la longeur d'onde d'excitation sêlective dans 1 a bande d'absorption $7 \mathrm{~F}_{0} \rightarrow{ }^{5} \mathrm{D}_{0}$. 
éọuidistantes. Au delà de l'excitation à $5777 \AA$ (d), les deux composantes de olus faible énergie s'êlargissente en présentant des épaulements de polus en plus marqués. La composante de plus forte énergie diminue lëgèrement d'intensité, mais reste fine. La bande correspondant à la transition ${ }^{5} D_{0} \rightarrow F_{2}$ subit également une évolution en fonction de la longueur d'onde d'excitation. Cependant, les variations observées sont moins caractéristiques que pour la transition $5 \mathrm{D}_{0} \rightarrow 7_{\mathrm{F}}$.

$L$ 'enregistrement des spectres d'émission, pour une énergie d'excitation constante $\left(\lambda_{\text {exc }}=5778 \AA\right)$ a été effectué à intervalles de temns variables (fig. 4). Ces spectres ne laissent apparâtre aucune variation sensible de la position des trois raies relatives à la transition $5 \mathrm{D}_{0} \rightarrow ? \mathrm{~F}_{1}$. On constate cependant, une évolution de l'intensité de celles-ci en fonction de la durée de l'observation. Le profil des rajes situées entre 5900 et $5950 \AA$ s'affine, tandis que celui à $5860 \AA$ ne subit aucune modification.

4.2. Interprétation et discussion. - La prësence simultanément de la transition $5 \mathrm{D}_{0} \rightarrow{ }_{\mathrm{F}}$ et de trois composantes STARK pour 1 a transition $5 \mathrm{D}_{0} \rightarrow 7 \mathrm{~F}_{1}$ permet de situer $7^{Q} i$ on Eu ${ }^{3+}$ dans un site de symétrie $C_{2 v}$. L'évolution du profil, de la résolution de ces composantes ainsi que de leurs éclatements en fonction de 1 'énergie d'excitation croissante laisse supposer que nous sommes en présence d'un passage progressif d'une famille de sites homogènes (site I) de symétrie $C_{2 v}$ à une autre famille possédant une plus large distribution (sites II), de même sumétrie mais avec forte distortion.

La taille de l'ion Eü est très voisine de celle de 1'ion $\mathrm{Ca}^{2+}$, mais heaucoup plus petite que celle de 7 'ion $\mathrm{Ba}^{2+}$. Il est donc possible que les deux familles de sites, I et II, proviennent respectivement de la substitution d'ions $\mathrm{Ca}^{2+}$ et d'ions Ba2+ par les ions Eu ${ }^{3+}$. Cette hypothèse pourrait être renforcée par le fait que dans la bande inhomogène ${ }^{5} D_{0} \rightarrow{ }^{7} F_{0}$, 1 'émission des ions Eu ${ }^{3+}$ dans les sites I s'effectue à une énergie plus fäble que celle des sites II:1a liaison Ca-F est effectivement moins ionique que la liaison Ba-F. Ces positions correspondent
d'ailleurs à celles déterminées par P. VALON et al dans BaCaLu2 $F_{10}$ où les ions $\mathrm{Eu}^{3+}$ occupent simultanément aussi les sites de calcium et du baryum $(5)$.

L'étude de 1 'émission résolue dans le temps pour une excitation commune des deux espèces de sites met en évidence un transfert d'ënergie non radiatif entre les deux families de sites (fig. 4). Nous n'observons en effet aucun déplacement de la position des composantes STARK. De plus, celles-ci s'homogénéisent pour des observations à des tamps longs. Ce transfert semble d'effectuer des sites les plus ioniques (sites II) vers les plus covalents (sites I). IT est très important, car nous n'observons qu'une très faible émission pour les jons Eu ${ }^{3+}$ en sites II.

5. Diffusion Raman. - L'étude a été entreprise sur les quatre compositions de verres regroupées sur le tableau I (6).

\begin{tabular}{|c|c|c|}
\hline$N^{\circ}$ & Compositions & $\mathrm{AT} / \mathrm{Be}$ \\
\hline 1 & 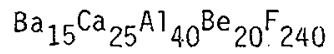 & 1. \\
\hline 2 & $\mathrm{Ba}_{15} \mathrm{Ca}_{25^{\mathrm{Al}}}{ }_{30} \mathrm{Be}_{30} \mathrm{~F}_{230}$ & 1 \\
\hline 3 & $\mathrm{Ba}_{15} \mathrm{Ca}_{20}{ }^{\mathrm{A}} \mathrm{l}_{25} \mathrm{Be}_{40} \mathrm{~F}_{225}$ & 0,62 \\
\hline 4 & $\mathrm{Ba}_{11} \mathrm{Ca}_{20} \mathrm{Al}_{23} \mathrm{Be}_{46} \mathrm{~F}_{223}$ & 0,5 \\
\hline
\end{tabular}

Tableau I - Composition des verres ABF ètudiés.

5.1. Résultats expérimentaux . - Les spectres correspondant aux polarisations paraillèle et croisée sont représentés sur la figure 5 . La présence de BeF 2 se manjfeste par une bande totalement nolarisée dont l'intensité et la fréquence croît et décroît respectivement avec sa teneur $\left(505 \mathrm{~cm}^{-1}, 495 \mathrm{~cm}^{-1}, 495 \mathrm{~cm}^{-1}\right.$, $490 \mathrm{~cm}^{-1}$ pour les verres $\left.1,2,3,4\right)$. Sur les flancs de cette large bande on note la présence de deux épaulements totalement polarisés à 620 et $560 \mathrm{~cm}^{-1}$. 

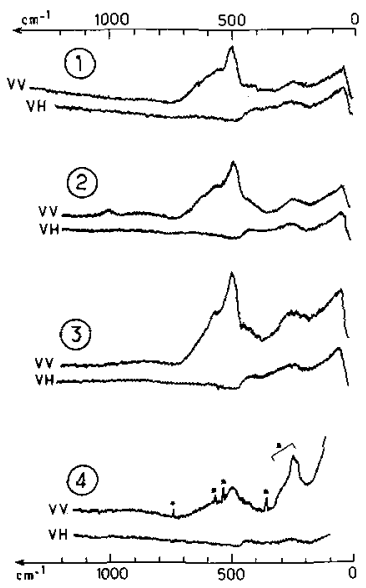

Fig. 5 - Spectres de diffusion Raman des verres $A B F$ 1-4. Partie supérieure spectre $V V$, partie inférieure spectre $V H$ * raies parasites (plasma laser).

5.2. Interprêtation et discussion. - Par comparaison avec la cryolithe (7) et les bains fondus d'alumino-fluorure (8), 1'épaulement à $560 \mathrm{~cm}^{-1}$ provient du mode totalement symétrique $v_{1}\left(\mathrm{AlF}_{6}^{3}\right)$. L'étude précédente de verres alumino-fiuorés (9) renforcée par la nature aciće du mijieu des verres ABF nous ont permis d'attribuer 1 'épaulement à $620 \mathrm{~cm}^{-1}$ à $v_{1}\left(A 1 \mathrm{~F}_{4}^{-}\right)$.

Les travaux sur des verres multicomposants riches en $B_{e} F_{2}(10,11)$, des bains fondus fluorobérytiés $(12,13)$ et les calculs de dynamique moléculaire ont permis d'admettre que d'une part les modes $v_{1}\left(A_{1}\right)$ et $v_{3}\left(A_{1}\right)$ des mono et dimères béryllês dominent toujours dans les spectres Raman, d'autre part, que la fréquence du mode décroît avec la polymérisation (tableau. II). Nous avons donc pu attribuer la bande

\begin{tabular}{lllc}
\hline $\operatorname{Modes}\left(\mathrm{cm}^{-1}\right)$ & \multicolumn{2}{c}{ NaF-LiF-BeF } & Phases cristal- \\
& Verre & Fonte & liseres \\
\hline$\nu_{1}\left(\mathrm{BeF}_{4}\right)^{2-}$ & $565^{\mathrm{b}}$ & $547^{\mathrm{a}}$ & $560^{\mathrm{a}}$ \\
$\nu_{3}\left(\mathrm{Be}_{2} \mathrm{~F}_{7}\right)^{3-}$ & $530^{\mathrm{b}}$ & $520^{\mathrm{b}}$ & $520^{\mathrm{b}}$ \\
\hline
\end{tabular}

Tableau II - Fréquence du mode $A_{1}$ le plus actif en diffusion Raman des especes $\mathrm{BeF}_{4}{ }^{2-}$ et $\mathrm{Be}_{2} \mathrm{~F}_{7}^{3-}$ dans diverses phases fluorobéry 17 ées

a d'après QUIST et al (12)

b d'après TOTH et al (13)

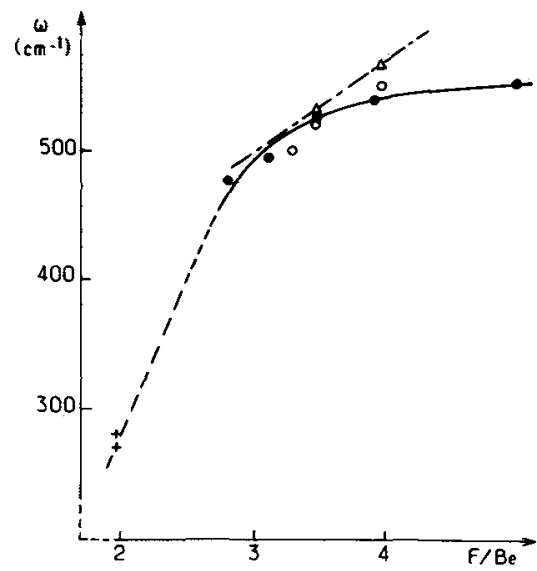

Figure 6 - Relations entre les fréquences de vibrations Be-F du mode symétrique le plus actif en diffusion Raman et le rapport atomique $\mathrm{F} / \mathrm{Be}$ pour divers composés fluorobéryllês

- bains fondus Li F-BeF $2(12,13)$

- entités Jibres $\left(\mathrm{BeF}_{4}\right)^{2}-\left(\mathrm{Be}_{2} \mathrm{~F}_{7}\right)^{3-}$ $\left(\mathrm{Be}_{3} \mathrm{~F}_{10}\right)^{4-}(13)$

$\Delta$ verres NaF-LiF-BeF 2 (13)

+ verre $\mathrm{BeF}_{2}(10,14)$. 
voisine de $500 \mathrm{~cm}^{-1}$ à des arrangements autour du bēryllium et de plus mesurer le degré moyen de polymérisation dans les verres ABF grâce à la courbe de la figure 6 . $\mathrm{Si}$ on néglige l'interaction avec les groupements aluminofluorés (pe $\mu$ de liaisons $\mathrm{A} 1-\mathrm{F}-\mathrm{Be})$ on peut en déduire la présence de polymères $\mathrm{Be}:\left(\mathrm{Be}_{n} \mathrm{~F}_{3 n+1}\right)^{(2+n)-}$. La valeur moyenne de l'indice $n$ serait voisine de 10 (tableau III, 3ème colonne). Dans ces conditions, compte tenu des atomes de fluor restant pour la coordination de l'aluminium, on peut également exclure l'existence d'un rêseau tridimensionnel d'octaèdres $\left(\mathrm{AlF}_{3}\right)$ (tableau III, 4ème colonne).

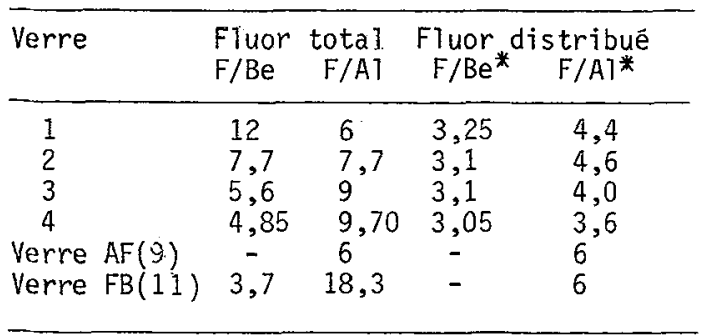

Tableau III - Evolution des compositions des verres $A B F$

* Valeurs limites en l'absence de liaisons mixtes Al-F-Be (voir texte).

En fait la présence d'aluminium tétracoordonné et très vraisemblablement l'existence des liaisons mixtes Al-F-Be ne peuvent qu'accroitre ces rapports qui deviennent alors compatibles avec $T$ 'existence d'enchainements d'octaèdres. On notera 1 'existence d'une faible bande polarisêe (spectre $\mathrm{n}^{\circ} 2$ ) vers $1000 \mathrm{~cm}^{-1}$ qui pourrait être attribuée à T'ion $\mathrm{Be}_{2} \mathrm{~F}_{7}^{3-}$ (13).

6. Hypothèse structurale. - Sur la base de nos études spectroscopiques, il est possible de formuler des hypothèses sur 1 'arrangement de ces divers polyèdres. Les tétraèdres $\mathrm{BeF}_{4} \mathrm{~s}^{\prime}$ associeraient par leurs sommets pour donner naissance, pour 1 'essentiel, à des chânes $\left(\mathrm{Be}_{1+n} \mathrm{~F}_{4+3 n}\right)(2+n)$. En principe, $n$ serait au moins égal à $5(n$ moyer $\sim 10)$. Il existerait cependant, en nombre limité, des entités pius courtes, consti tuées en général de deux tétraèdres $\left[\mathrm{Be}_{2} \mathrm{~F}_{7}\right]$. Par analogie avec les hypothèses formulées lors de 1 'étude des verres fluoroaluminés ( 9 ), que 1 'aluminium forme également des chaînes d'octaèdres $\left.(A]_{1+n} F_{6+5 n}\right)(3+2 n)$ - . Dans ces conditions, $i 1$ est possible que les tétraèdres $\left(A 1 F_{4}\right)$ assurent $?$ 'interconnection des deux types de chaines - fluorobéryllées et fluoroaluminées - qui se dëvelopperaient ainsi tridimensionnellement.

Le calcium (environnement octaédrique déformé) et le baryum (cooruinence au moins supërieure à 8) seraient situês hors de ce squelette "formateur". Le calcium se placerait dans des sites préférentiels, bien isolés les uns des autres, comme le montre 1 'étude spectroscopique. Le baryum, bien que distribué de façon plus aléatoire que le calcium, serait relativement proche de ce dernier, comme le laissent supposer les transferts d'énergie qui s'effectuent entre ces deux sites.

Ces verres sont donc très diffërents des matëriaux vitreux riches en fluorure de bëryliium dont le rëseau est formé tridimensionnellement des tētraèdres, mettant en commun la plupart de leurs sommets. Une telle conclusion est confirmée par les observations suivantes :

- dans les verres de type $A B F$, comme ceux que nous avons étudiés, le rapport $A 1 / B e$ doit être supérieur à 0,5 environ pour que ces verres puissent se former

- en revanche, dans les verres BF classiques (fluorobéryllés), ce rapport doit être inférieur à 0,5 environ. Pour les premiers de ces matériaux $(A \mathrm{Al} / \mathrm{Be}>0,5)$ si on ajoute au liquide en fusion un excès de fluorure de béryllium, on constate 1'apparition de phases cristallisées. Ce phénomène se produit au contraire pour les seconds $(A 1 / B e<0,5)$ lorsque 1 'on ajoute un excès de fluorure d'aluminium. Il y a donc incompatibilité entre les deux types de matériaux, dont les structures sont nécessairement différentes.

\section{Remerciements :}

Les auteurs remercient.MM. BLANZAT, BARTHOU et DANCE pour leur coopération durant les mesures spectroscopiques et les fructueuses discussions. 


\section{RÉFÉRENCES}

(1) VIDEAU J.J., FAVA J., FOUASSIER F. et HAGENMULLER P., Mat. Res. Bu11., 14 (1979) 499.

(2) LOVERIDGE D. et PARKE S, Phys. Chem. of Glasses, 12(1971) 19.

(3) GRISCOM D.L. et GRISCOM R.E., J. Chem. Phys., 47(1967) 2711.

(4) VIDEAU J.J., PORTIER J. BLANZAT B et BARTHOU C., Mat. Res. Bu11., 14(1979)1225.

(5) VALON P. GACON J.C., VEDRINE A. et BOULON G., J. Solid State Chem., 21 (1977) 357.

(6) PIRIOU B., VIDEAU J.J. et PORTIER J., J. Non Cryst. Solids, 46 (1981) 105.

(7) WICKOFF A.W.G., Cryst. Struct., 3 (1965) 382.

(8) GILBERT B. et MAMANTOV G. J. Chem. Phys., 62 (1975) 950.

(9) VIDEAU J.J., PORTIER J. et PIRIOU B., Rev. Chim. Min. 16 (1979) 393.

(10) WALRAFEN G.E. et STOLEN R.H., Sol. St. Commun., 21 (1977) 417.

(11) LAYNE C.B. et WEBER M.J., Phys. Rev., 16B (1977) 3259.

(12) QUIST S. BATES J.B. et BOYD G.E., J. Phys. Chem., 76 (1972) 7819.

(13) TOTH C.M., BATES J.B. et BOYD G.E., J. Phys. Chem., 77 (1973) 216.

(14) BATES J.B., J. Chem. Phys. 56 (1972) 1910. 